An evaluation of the potential of Web 2.0 API's for social research

\title{
Peter Mechant
}

IBBT-MICT-Ghent University, Department of Communication Sciences

Korte Meer 7-9-11

9000 Ghent, Belgium

Peter.Mechant@Ugent.be

Tel: +3292649708

\section{Cédric Courtois}

IBBT-MICT-Ghent University, Department of Communication Sciences

Korte Meer 7-9-11

9000 Ghent, Belgium

Cedric.Courtois@Ugent.be

Tel: +3292649154 
Web 2.0 not only changed the role of internet users (from consumer to prosumer or produser), it also changed the architecture and structure of websites. New scripting languages such as Ajax (Asynchronous Javascript and $\mathrm{XML}$ ), new software architectures such as REST (Representational State Transfer) and API's (Application Programming Interfaces) as gateways, short-circuited the submit/response loop that dominated web pages until recently, making data easier to share, verify and re-use. Nowadays, (amateur) programmers, web developers, creators of third-party applications and scientists can access Web 2.0 sites through API's - sets of rules and specifications for interacting with websites - as if they were data servers with multiple front ends.

This paper presents a reflection on the potential of these API's as exclusive or additional data resources for research into the practices of internet users. A literature review is presented that critically summarises articles using the Flickr or YouTube API for online audience research. We especially focus on how the use of Web 2.0 affordances (such as tagging, rating or commenting) can be investigated.

Although we distinguish a wide variety of research threads (e.g. on the features of the network structure, the user sessions or on the folksonomy and tagging system) we notice that little is known about the predominant practices of 'ordinary' Web 2.0 users. Furthermore, results are difficult to compare because of website specific features. We also conclude that research combining API data with qualitative or quantitative data gathered through surveys or interviews is rather sparse. 